\title{
ROLE AND FUNCTION OF A HISTORICAL NOVEL IN A POST COLONIAL SOCIETY
}

\section{Cynthia McLeod ${ }^{1}$}

"In a nation where the people have no access to the sources of its history, the self image will be based on mythes and stereotypes". This was one of the statements of the famous historian Barbara Tuchman in her PHD "A distant Mirror", in 1978.

When I read this as a young woman, in the late 70's of last century, I could only agree and realize that this was what had happened with the people of Surinam. We had no access to the sources of our history and we really developed a self-image based on myths and stereotypes. Archives, written material, about our three hundred years history were stationed in Holland and in Suriname one could only find information of the last 80 years.

Let me first tell you something about Suriname, which is Brazil's small neighbor in the North and the youngest country on the South American continent. In this former Dutch colony, the modern population consists of descendants of Africans who were brought as slaves and people from other continents, just like many other Caribbean countries. Transatlantic slavery and indentured labor had brought them to this part of the world where Dutch and other slave masters had a very harsh reign with many rules of what to do and what was forbidden for slaves. Unlike other countries where the enslaved had to adapt to the religion and the language of their masters, in Suriname slaves could NOT become Christians and were not permitted to speak or even know Dutch.

The 300.000 enslaved Africans who were brought to the country had a diversity of mothertongues and in Suriname a language developed which was spoken amongst them; first as pidgin but gradually this language became the mother tongue of the slaves born in Suriname. This language had African elements and included many words of the masters languages like English, Portuguese, Dutch. It became a creole language which was generally spoken and was the communication language between slave and master. The official language of the colony was Dutch, but the creole language developed very well, first it was called negre, (What means negro) now the name is Sranan tongo or Sranan (language of Suriname). It is the national language but not the official language. The official language is still Dutch.

Thanks to liberators as Simon Bolivar, the majority of the Latin American countries could free themselves from Spain and Portugal already in early eighteenhundreds. They became independent countries and developed their own culture and an own educational system although the language stayed Spanish and Portuguese. In Ecuador and Peru for instance the majority of the population speaks Kechua at home, but the education is in Spanish. Suriname was a Dutch colony and just like in the rest of this continent, the language of the former colonizer became our official language, what gave Suriname also an exceptional place in this continent, namely the one and only Dutch speaking country on the western hemisphere.

Emancipation of slavery was in 1863 and right after that, the country got compulsory education for all children between 6 and 12. This education was a copy of the Dutch education, all children had to go to school and had to speak Dutch from one day to the other, never mind that the former enslaved didn't speak Dutch.

Suriname was a plantation colony, nothing else and the owner of the colony had to find workers for the plantations, that's why after slavery time, Holland imported indentured labors from China, than from British India, and Java (Indonesia). Just accidentally some more came from Lebanon and Madeira. Although many of them kept their mother tongue at home, the first

\footnotetext{
${ }^{1}$ Surinamese writer. E-mail: cynthia.mcleod.ferrier@gmail.com
} 
language they learned was Sranan tongo for communication in the street, in business. But their children had to go to school and had to speak Dutch. In school Sranan tongo was forbidden. All parents wanted their children to develop, no more slave labor, no more plantation labor, but a good job and to get that, you had to be educated, so speak Dutch, even at home it became a rule: speak Dutch. In school and sometimes even at home, children were severely punished if they dared to speak Sranan tongo.

In the Dutch school system in Suriname, children hardly learned anything about their country; all books came from Holland, Dutch textbooks, but also geography, history, science were about the Netherlands. So, in the tropical heat of Suriname children recited poems about snow and wrote essays about ice skating; they knew about all the wars in Europe but they knew nothing about Marrons and indigenous people in Suriname. But most of all, nothing of Suriname had any value. Norms and values were Dutch and every Surinamese element was inferior. Speaking Surinamese or Sranan tongo was forbidden, Surinamese music was inferior, Surinamese dancing was wrong, not decent. Although the majority of the schoolchildren had enslaved ancestors, they practically learned nothing about slavery time and whenever slavery was the subject, all they heard was that all colored people had to be so grateful that slavery was abolished. In their text books they could only read about children in Holland or elsewhere in Europe, so Surinamese children had a pretty good idea how it was to be a poor child in an European city, but they could not imagine how it must have been to be a slave child.

In the homely atmosphere sometimes they could hear stories, there were folk songs and verbs dating from slavery time, but these had no value, no status. As you all know, during the 20th century, the whole world experienced every day the white West European and American White supremacy. There was the place where one had civilization and culture. What we had in Suriname was no culture at al. For the common Surinamer, no matter what background he had creole, or Hindu, Chinese or Javanese, everyone should realize that Dutch and Holland was the role model, everyone had to become as Dutch as possible and the utmost was to go to Holland yourself and become part of this superb civilization and culture.

They went! After World War II many young Surinamers went to Holland for higher education. Even in the poorest family, parents worked hard to save some money to buy at least the ticket for Holland. Being there, the majority of these Surinamese students had a big culture shock. Because they realized that they had a Dutch education but they themselves were not Dutch and would never be considered to be Dutch; they had an own Surinamese identity and they realized that there was nothing wrong with having a Surinamese identity. They started to behave different from all that Dutch what was enforced on them. They spoke Sranan tongo, they made Surinamese music, they sang all the in Suriname forbidden songs, they had Surinamese parties where they danced their own way and of course they ate Surinamese food! The majority of Surinamese music orchestras and bands started in Holland. And they enjoyed.

Many of these first students returned and back in Suriname, being doctor or lawyer anyhow with a high education, they became leaders in social life, in business in politics. They became role models and the rest of the people looked up to them, but these highly educated people spoke Sranan tongo, they proudly enjoyed Surinamese music and songs. This was good and that's how Surinamers started to value their own things and also became opponents against everything that was so ultraDutch. And this is also how the population became more and more interested to become an independent country, what happened in 1975. We didn't fight or struggle though to become independent, the Netherlands was happy to get rid of us.

I myself, I am one of these students from the late fifties and sixties, I experienced this all. As a child in Suriname in school, I really missed it that I learned nothing about our own background. Of course I knew that some of my ancestors had been slaves but I never heard anything about them, my maternal grandparents had both Portuguese Jewish names, so I knew that somewhere along the lines I had Jewish forefathers and I knew that my great grandmother came from India and often I heard about her youth and what had happened to her, but about the 
slave ancestors I knew nothing. I started to research myself, but this was practically impossible in Suriname because everything was in Holland.

Four to five generations of speaking Dutch and the Dutch education had a result that this language became the mother tongue of the majority, although in many diverse ethnic groups, the home language is the ethnic language. All Surinamese children are at least bi-lingual and Sranan tongo is a vivid language known by everyone, now considered as the national language of Suriname. Independence was in 1975. With this small population and wide diversity of background and languages, it was the best to stay with Dutch as the official language and the Dutch education. Textbooks are in Dutch, but in elementary education our children read stories about Surinamese children playing in the back yard and in geography class they learn about Surinamese rivers and mountains and the Amazon region.

I myself was a college professor in the seventies, not history, but Dutch and Literature. With colleagues we changed the curriculum for Literature from totally Dutch and European into Dutch, Latin American, Caribbean and Surinamese. And we introduced a subject: Surinamese Literature. There was nothing. As a professor, you had to do research yourself and I did. I found, in old newspapers, in records of churches. My students were teenagers, young adults and I discovered that my students hardly knew anything about Surinamese history and what they knew were stories. But stories are very often myths and stereotypes. I often tried to undo the myths and stereotypes and told about what really happened,how the reality had been, how life had been in slavery time. They were really interested, they wanted to hear more and more and they were the ones who encouraged me tremendously to write and become an author. I often told them that I would when I was retired. Fate was on my side, after independence my husband became an Ambassador and I had time to write, we were sent to Brussels, so near Holland and The Hague where the written sources of our history were, the Dutch National Archives. I did, I was so often in the Archives that even the people of the Archives got to assume that I was working there.

I chose to write novels, historical novels and I really gave myself the assignment to go for the truth, I could fantasize and be creative in the emotions of my personalities but never in historical facts. These historical facts in my novels very often had a totally different perspective than how I found them in the archives, because they were written down from the perspective of the colonizer, but now I enlighted them from the Surinamese side but I never changed the facts.

My first historical novel was published in 1987, now 30 years ago. It became a real hype and it still is the nr 1 best seller in Suriname. Within 3 weeks all the books were sold, it had more than 30 reprints, it was filmed and translated in English, German, Italian; it's to be heard on DVD. Very often people wonder why this book became so special. I know the answer: This was the very first book about Surinamers own background, written in an easy way. Oral history, myths are nice but people also want to know the truth in their history. It might be different than the myth and the oral history but people are also eager to know the truth. A Dutch newspaper in Holland wrote that this first Surinamese historical novel was of immense value. I decided to research more and write more, especially about important issues as uproars, and the facts which came in history books in a total different way than how it really was, like my favorite: The free and very rich Negress Elisabeth, of whom the history writers had written that she had inherited her wealth from a white man whose slave and concubine she had been, while I could proof with facts and archive material that she had never inherit from a white man, she had made her wealth herself and in contrary, she made white families rich. The only thing what the history writers had proofed with their assumption was their machoism and white supremacy. But this is a story for another time.

\section{References}

TUCHMAN, Barbara. A Distant Mirror: the Calamitous 14th Century. London: Random house trade, 1987. 


\title{
O PAPEL E A FUNÇÃO DO ROMANCE HISTÓRICO EM UMA SOCIEDADE PÓS-COLONIAL ${ }^{2}$
}

\author{
Cynthia McLeod ${ }^{3}$
}

"Em uma nação onde as pessoas não têm acesso às fontes de sua história, a autoimagem será baseada em mitos e estereótipos". Esta foi uma das afirmações da famosa historiadora Barbara Tuchman em seu PHD "Um espelho distante", em 1978.

Quando li esse trecho -enquanto uma jovem mulher, no final dos anos 70 do século passado -, eu só pude concordar e perceber que isso era o que havia acontecido com o povo do Suriname. Não tínhamos acesso às fontes da nossa história e realmente desenvolvemos uma autoimagem baseada em mitos e estereótipos. Arquivos e material escrito sobre a nossa história de trezentos anos estavam esquecidos na Holanda e no Suriname e, além disso, só era possível encontrar informações dos últimos 80 anos.

Deixe-me primeiro falar sobre o Suriname, que é o pequeno país vizinho ao norte do Brasil e o mais jovem do continente sul-americano. Nessa antiga colônia holandesa, a população moderna consiste de descendentes de africanos que foram escravizados e de pessoas de outros continentes, assim como em muitos outros países caribenhos. O trabalho escravo levou-nos a esta parte do mundo onde os holandeses e outros senhores de escravizados eram muito duros e tinham muitas regras sobre o que era permitido e o que era proibido. Ao contrário de outros países, onde os escravizados tinham que se adaptar à religião e à língua de seus senhores, no Suriname, eles NÃO podiam se tornar cristãos e não tinham permissão para falar ou mesmo conhecer o holandês.

Os 300.000 africanos escravizados que foram trazidos para o país falavam diversas línguas e, no Suriname, uma foi desenvolvida a partir dessas: primeiro como pidginque, gradualmente, tornou-se a língua materna dos escravos nascidos no Suriname. Esta língua tinha elementos africanos e incluía muitas palavras das línguas dos colonizadores como o inglês, o português, o holandês. Tornou-se uma língua crioula falada por todos e era o idioma de comunicação entre o escravizado e o mestre. Assim, a língua oficial da colônia era a holandesa, contudo, o crioulo passou a ter maior adesão. Primeiramente, ele foi chamado de negre (o que significa negro), agora seu nome é Sranan Tongo ou Sranan (língua do Suriname). É a língua nacional do país, mas não a oficial, que continua sendo a holandesa.

Graças a lideranças como Simón Bolívar, a maioria dos países latino-americanos pôde se libertar da Espanha e de Portugal já no início do século XIX. Ao se tornarem independentes, desenvolveram cultura e um sistema educacional próprios, embora a língua permanecesse espanhola e portuguesa. No Equador e no Peru, por exemplo, a maioria da população fala kechua em casa, mas a educação é em espanhol. O Suriname era uma colônia holandesa e, assim como no restante do continente, a língua do ex-colonizador tornou-se nossa língua oficial, o que deu ao Suriname um lugar excepcional neste continente, o único país de fala holandesa no hemisfério ocidental.

A emancipação da escravidão foi em 1863 e, logo em seguida, a educação se tornou obrigatória para todas as crianças entre 6 a 12 anos. Ela foi uma cópia da educação holandesa e, então, todas as crianças tiveram que falar holandês de um dia para o outro sem considerar que o ex-

\footnotetext{
2 Tradução de Natali Fabiana da Costa e Silva. Doutora em Estudos Literários e Professora de Teoria Literária da Universidade Federal do Amapá. E-mail: natali_costa@hotmail.com

3 Surinamese writer. E-mail: cynthia.mcleod.ferrier@gmail.com
} 
escravizado não falavaesse idioma.

O Suriname era uma colônia de plantações, nada mais, e o dono da colônia tinha que encontrar trabalhadores para as plantações, por isso, depois da escravidão, a Holanda importava mão de obra da China, da Índia Britânica e de Java (Indonésia). Apenas acidentalmente mais alguns vieram do Líbano e da Madeira. Embora muitos deles mantivessem sua língua materna em casa, a primeira língua que aprenderam foi oSranan Tongo para comunicação na rua, nos negócios. Mas seus filhos tiveram que ir à escola e tiveram que falar holandês. Na escola, Sranan Tongo foi proibido. Todos os pais queriam que seus filhos fossem bem sucedidos, que não trabalhassem mais como escravizados, que não trabalhassem mais em plantações, mas que tivessem um bom emprego. Para conseguir isso, seria necessário ser educado e, então, falar holandês - mesmo dentro de casa - tornou-se uma regra. Na escola e, às vezes, até em casa, as crianças eram severamente punidas se ousassem falar em Sranan Tongo.

No sistema escolar holandês no Suriname, as crianças quase não aprendiam sobre seu país, pois todos os livros vinham da Holanda. Livros didáticos holandeses e também livros de geografia, de história e de ciência falavam todos sobre a Holanda. Assim, no calor tropical do Suriname, crianças recitavam poemas sobre a neve e escreviam ensaios sobre patinação no gelo; elas sabiam sobre todas as guerras na Europa, mas não sabiam nada sobre os marrons e os povos indígenas do Suriname. Mas acima de tudo, nada do Suriname tinha valor: normas e valores eram holandeses e todos os elementos surinameses eram inferiores. Falar Surinamês ou Sranan Tongo era proibido, a música surinamesa era inferior, a dança surinamesa era errada, não era decente. Embora a maioria dos alunos tivesse ancestrais que haviam sido escravizados, eles praticamente não aprendiam nada sobre o tempo da escravidão e sempre que isso era assunto, tudo o que ouviam era somente que tinham que agradecer à abolição da escravidão. Nos livros didáticos, lia-se sobre crianças na Holanda ou em outros lugares da Europa, então as crianças surinamesas tinham uma boa ideia de como era ser uma criança pobre em uma cidade europeia, mas não podiam imaginar como era a vida de uma criança escravizada.

Em casa, por vezes, ouviam-se histórias ou canções folclóricas datadas do tempo da escravidão, mas estas não tinham nem valor nem status. Como sabemos, durante o século XX, todos os dias o mundo inteiro experimentou a supremacia branca da Europa Ocidental e dos Estados Unidos da América. Lá era o lugar da civilização e da cultura. O que tínhamos no Suriname não era cultura alguma. Para o Surinamês comum, não importava qual idioma ele conhecia: crioulo, hindu, chinês ou javanês, todos deveriam perceber que o holandês e a Holanda eram o modelo, todos deveriam se tornar tão holandeses quanto possível. Era o máximo ir à Holanda e fazer parte dessa civilização e cultura.

E eles foram! Depois da Segunda Guerra Mundial, muitos jovens surinameses foram para a Holanda a fim de cursar o ensino superior. Mesmo nas famílias mais pobres, os pais trabalhavam arduamente para economizarem algum dinheiro e comprarem, ao menos, a passagem para a Holanda. Chegando lá, a maioria desses estudantes surinameses experimentava um grande choque cultural porque percebiam que a despeito da educação holandesa, eles próprios não eram holandeses e nunca seriam considerados como tais; eles tinham uma identidade surinamesa própria e perceberam que não havia nada de errado nisso. Começaram a se comportar de maneira diferente de todo aquele holandês imposto a eles. Falavam Sranan tongo, faziam música surinamesa, cantavam todas as canções proibidas do Suriname, faziam festas surinamesas e, claro, comiam comida surinamesa! A maior parte das orquestras e bandas de música surinamesas começou na Holanda. Muitos desses primeiros estudantes voltaram ao Suriname como médicos ou advogados, tornaram-se líderes na vida social, nos negócios, na política. Eles se tornaram exemplos e o resto do povo olhava para essas pessoas altamente educadas que falavam Sranan tongo e desfrutavam orgulhosamente da música e das canções surinamesas. Isso era bom e foi assim que os Surinameses começaram a valorizar sua própria cultura e também a se opor a tudo que era ultra holandês. Também foi assim que a população ficou mais e mais desejosa de se tornar um país independente, o que aconteceu em 1975. Nós não lutamos para nos tornarmos independen- 
tes, a Holanda ficou feliz em se livrar de nós.

Eu mesma sou uma entre esses alunos do final dos anos cinquenta e sessenta. Eu experimentei tudo isso. Quando criança, realmente sentia falta de não ter aprendido nada na escola sobre nossa própria história. Evidentemente, sabia que alguns dos meus ancestrais haviam sido escravizados, mas nunca ouvi nada sobre eles. Meus avós maternos tinham nome judaico-português, então sabia que tinha antepassados judeus e sabia também que minha bisavó viera da Índia, pois muitas vezes eu ouvi sobre sua juventude e sobre o que havia acontecido com ela. Contudo, sobre os ancestrais escravizados eu não sabia nada. Comecei a pesquisar sobre mim, mas isso era praticamente impossível no Suriname, pois tudo estava na Holanda.

Quatro ou cinco gerações de falantes holandeses fizeram com que o holandês se tornasse a língua materna da maioria, embora em diversos grupos étnicos, a língua materna seja a língua étnica. Todas as crianças surinamesas são bilíngues, o Sranan tongo é uma língua viva e conhecida por todos e, agora, considerada a língua nacional do Suriname. A independência foi em 1975. Com esta pequena população e com a grande diversidade de backgrounds e de idiomas, foi melhor adotar o holandês como língua oficial, assim como a educação holandesa. Os livros didáticos estão em holandês, mas no ensino fundamental, nossas crianças leem histórias sobre crianças surinamesas brincando no quintal, e na aula de geografia aprendem sobre os rios e montanhas do Suriname e sobre a região amazônica.

Eu mesma fui professora universitária nos anos 70, não de história, mas de holandês e de literatura. Com os colegas, mudamos o currículo da Literatura que era holandesa e europeia para holandesa, latino-americana, caribenha e surinamesa. E nós introduzimos um assunto: Literatura Surinamesa. Não havia nada. Como professor, você tem que fazer pesquisa e, então, eu fiz. Busquei em jornais antigos, em registros de igrejas. Meus alunos eram adolescentes e adultos e descobri que eles mal sabiam alguma coisa sobre a História surinamesa e o que eles sabiam eram apenas histórias. Mas histórias são muitas vezes mitos e estereótipos. Muitas vezes, tentei desfazer esses mitos e estereótipos e contei sobre o que realmente aconteceu, como havia sido arealidade, como a vida tinha sido no tempo da escravidão. Eles ficavam realmente interessados, queriam ouvir mais e mais e foram eles que me encorajaram a escrever e a me tornar uma escritora. Costumava dizer-lhes que eu escreveria quando me aposentasse. O destino estava do meu lado: depois da independência, meu marido se tornou embaixador e eu tive tempo de escrever. Fomos enviados para Bruxelas, tão perto de Haia, onde estavam as fontes escritas de nossa história, nos Arquivos Nacionais da Holanda. Eu escrevi, estava tão frequentemente nos Arquivos que até mesmo alguns funcionários assumiram que eu trabalhava ali.

Escolhi escrever romances - romances históricos - e realmente me dei a missão de buscar a verdade, eu poderia fantasiar e ser criativa nas emoções das minhas personagens, mas nunca em fatos históricos. Esses fatos históricos em meus romances muitas vezes tinham uma perspectiva totalmente diferente de como os encontrei nos arquivos, pois foram escritos sob a perspectiva do colonizador, mas agora eu os iluminava pela perspectiva do surinamês (mas nunca mudei os fatos).

Meu primeiro romance histórico foi publicado em 1987, há 30 anos. Tornou-se um verdadeiro sucesso e ainda é o número $1 \mathrm{em}$ vendas no Suriname. Dentro de três semanas, todos os livros haviam sido vendidos e ele teve mais de 30 reimpressões, foi filmado e traduzido em inglês, alemão e italiano. Muitas vezes as pessoas se perguntam por que esse livro se tornou tão especial. Eu sei a resposta: este foi o primeiro livro sobre a própria origem do Suriname, escrito de uma maneira fácil. História oral e mitos são bons, mas as pessoas também querem saber a verdade em sua história. As pessoas estão ansiosas para saber a verdade. Um jornal na Holanda escreveu que este primeiro romance histórico surinamês foi de imenso valor. Decidi pesquisar mais e escrever mais, especialmente sobre questões importantes como revoltas [BALBÚRDIA] ${ }^{4}$. Os fatos que

${ }_{4}^{4}$ Termo consentido pela autora para a tradução do português.

https://periodicos.unifap.br/index.php/letras

Macapá, v. 9, n. 2, $2^{\circ}$ sem., 2019 
estavam nos livros de história vinham agora de um modo totalmente diferente, como no meu livro favorito: The free and very rich Negress Elisabeth. Dela, os historiadores haviam falado que herdara sua fortuna de um homem branco, pois havia sido sua escrava e concubina. Eu pude provar com fatos e material de arquivo que ela nunca herdou nada de um homem branco, pois ela não somente fez sua riqueza como, ainda, tornou famílias brancas ricas. A única coisa que os escritores de história haviam provado com sua suposição era o machismo e a supremacia branca. Mas esta é uma história para outra hora.

\section{Referências}

TUCHMAN, Barbara. A Distant Mirror: the Calamitous 14th Century. London: Random house trade, 1987.

Envio: 30/10/2019

Aceite: 08/11/2019 